\title{
An Adaptive Observer-Based Controller Design for Active Damping of a DC Network With a Constant Power Load
}

\author{
Juan E. Machado ${ }^{\circledR}$, Romeo Ortega, Alessandro Astolfi ${ }^{\circledR}$, Fellow, IEEE, José Arocas-Pérez ${ }^{(}$, \\ Anton Pyrkin $^{\circledR}$, Member, IEEE, Alexey A. Bobtsov ${ }^{\circledR}$, Senior Member, IEEE, \\ and Robert Griñó ${ }^{\circledR}$, Senior Member, IEEE
}

\begin{abstract}
This article explores a nonlinear, adaptive controller aimed at increasing the stability margin of a direct-current (dc), small-scale, electrical network containing an unknown constant power load (CPL). Due to its negative incremental impedance, this load reduces the effective damping of the network, which may lead to voltage oscillations and even to voltage collapse. To overcome this drawback, we consider the incorporation of a controlled dc-de power converter in parallel with the CPL. The design of the control law for the converter is particularly challenging due to the existence of unmeasured states and unknown parameters. We propose a standard input-output linearization stage, to which a suitably tailored adaptive observer
\end{abstract}

Manuscript received May 25, 2020; revised September 10, 2020; accepted November 6, 2020. Manuscript received in final form November 10, 2020. The work of Juan E. Machado was supported by the National Council of Science and Technology (CONACyT), Mexico. The work of Romeo Ortega was supported in part by NSFC under Grant 61473183 and Grant U1509211. The work of Alessandro Astolfi was supported in part by the European Union's Horizon 2020 Research and Innovation Programme under Grant 739551 (KIOS CoE). The work of José Arocas-Pérez and Robert Griñó was supported in part by the Government of Spain through the Agencia Estatal de Investigacion under Project DPI2017-85404-P and in part by the Generalitat de Catalunya under Project 2017 SGR 872. The work of Anton Pyrkin and Alexey A. Bobtsov was supported by the Ministry of Science and Higher Education of the Russian Federation (Creation of high-tech production of robotic flaw detectors for monitoring hard-to-reach welded joints and metal structures of hazardous production facilities in industry, energy, and housing) under Grant 074 11-2018-029, 13.07.2018. Recommended by Associate Editor J. B. Hoagg. (Corresponding author: Juan E. Machado.)

Juan E. Machado is with the Discrete Technology and Production Automation (DTPA), University of Groningen, 9747 AG Groningen, The Netherlands (e-mail: juan.eduardo88@gmail.com).

Romeo Ortega is with the Departamento Académico de Sistemas Digitales, Instituto Tecnológico Autónomo de México (ITAM), Mexico City 01080, Mexico, and also with the Department of Computer Science and Control Systems, ITMO University, 197101 Saint Petersburg, Russia (e-mail: romeo.ortega@itam.mx).

Alessandro Astolfi is with the Department of Electrical and Electronic Engineering, Imperial College London, London SW7 2AZ, U.K., and also with the DICII, Università di Roma "Tor Vergata," 00133 Rome, Italy (e-mail: a.astolfi@imperial.ac.uk).

José Arocas-Pérez and Robert Griñó are with the Institute of Industrial and Control Engineering (IOC), Universitat Politècnica de Catalunya, 08028 Barcelona, Spain (e-mail: jose.arocas@upc.edu; roberto.grino@upc.edu).

Anton Pyrkin is with the Department of Computer Science and Control Systems, ITMO University, 197101 Saint Petersburg, Russia, and also with the Center for Technologies in Robotics and Mechatronics Components, Innopolis University, 420500 Innopolis, Russia (e-mail: a.pyrkin@gmail.com).

Alexey A. Bobtsov is with the Department of Computer Science and Control Systems, ITMO University, 197101 Saint Petersburg, Russia (e-mail: bobtsov@mail.ru).

Color versions of one or more figures in this article are available at https://doi.org/10.1109/TCST.2020.3037859.

Digital Object Identifier 10.1109/TCST.2020.3037859 is added. The good performance of the controller is validated through experiments on a small-scale network.

Index Terms-Active damping, adaptive control, adaptive observers, constant power loads (CPLs).

\section{INTRODUCTION}

$\mathbf{T}$ HIS note deals with the stabilization problem for electrical networks with constant power loads (CPLs). It is well known that, due to their negative incremental impedance, CPLs induce voltage oscillations or even voltage collapse [5]. The analysis of networks with this type of loads started in [11] and has been an active research area since (see [1], [2], [5]).

Various techniques have been explored for the stabilization of direct-current (dc) networks with CPLs-a survey may be found in [14]. These techniques are categorized into passive and active damping methods: the former is based on open-loop hardware alterations, whereas the latter imply the modification of existing — or added - control loops. In an active damping strategy, the control loops can be modified at three different network's positions [14]: at the source's side, the load's side, and a midpoint between them. In this article, we are interested in the latter approach, which was first explored in [3], [16], and [8], for the stabilization of a small-scale network with a single CPL. In these references, the network's stabilization is achieved by adding a controlled power converter in parallel with the load and then designing a suitable feedback control law for it. In [3], the converter is modeled as a simple controlled current source and a linear control law is designed to stabilize the overall network. A similar approach, but using a full model for the power converter, is used in [16]. Their stabilization result is based on the linearization of the network's dynamics. Finally, in [8], a large-signal stability analysis, but using approximate techniques, such as the Takagi-Sugeno fuzzy model, is carried out to evaluate the performance of a linear controller.

The main contribution of this article is described next. Following [3] and [16], we study the stabilization problem for a small-scale dc network supplying electrical energy to a CPL. First, we augment the network by placing a controlled power converter between the load and the source. Then, for the converter's controller design, instead of relying on linear-feedback techniques, we propose an adaptive observerbased nonlinear control law that provably achieves overall 


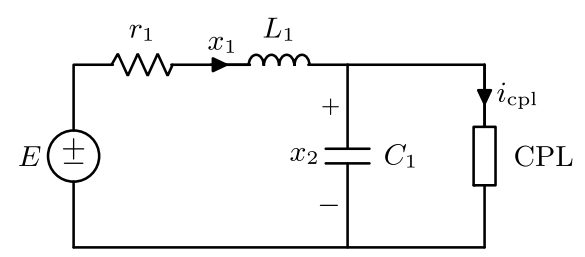

Fig. 1. DC source supplying power to an instantaneous CPL.

network's stabilization. The control design is particularly challenging due to the existence of unmeasured states - the current of the dc network-and the unknown power of the CPL. The construction of the proposed controller is based on the use of standard input-output linearization to which a suitably tailored adaptive observer is added; its good performance is evaluated via experiments on a small-scale dc network.

The stabilization problem addressed in this article, as well as the proposed controller topology, have previously been studied in [10], where a full state-feedback adaptive passivity-based control has been proposed. As discussed in Section IV-A, besides the impractical requirement of full state measurement, the approach adopted in that paper suffers from significant energy efficiency drawbacks, which renders the proposed controller design practically unfeasible. Both limitations are overcome in this article.

The rest of this article is structured as follows. In Section II, we present the model of the system under study and summarize its stability properties. The proposed controller configuration, adopted from [3] and [16], is presented in Section III. The main stabilization results are presented in Section IV. Our results are first illustrated with some simulations in Section V, and in Section VI, we present experimental evidence of the successful operation of the proposed control scheme. This article is wrapped-up with a number of concluding remarks in Section VII.

Caveat Emptor: A preliminary version of this work has been reported in the conference paper [9]. In contrast to [9], in the present work, we include the proofs of all propositions and add more detailed simulations and experimental results.

\section{PROBlem Formulation}

\section{A. Description of the System Without the Shunt Damper}

The circuit diagram of the electrical network under study is shown in Fig. 1. It represents a simplified model of a dc power system and has been used in the literature, e.g., in [12], [15], and [16], to study the stability problems associated with CPLs. It is composed of a dc voltage source supplying energy to an instantaneous CPL. The transmission line is simply represented by the lossy inductor $L_{1}>0$ and the CPL is connected through the bus capacitor $C_{1}>0$. The network's dynamics are described by

$$
\begin{aligned}
& L_{1} \dot{x}_{1}=-r_{1} x_{1}-x_{2}+E \\
& C_{1} \dot{x}_{2}=x_{1}-\frac{P}{x_{2}}
\end{aligned}
$$

where $x_{1}$ and $x_{2}$ denote the current through $L_{1}$ and the voltage across $C_{1}$, respectively. The constant parameter $P$ corresponds to the power extracted from, or injected to, the network by the CPL, being positive in the former case and negative in the latter-in the sequel, we focus our attention on the case $P \geq 0$. The state space for this system is defined as the set

$$
\left\{\left(x_{1}, x_{2}\right) \in \mathbb{R}^{2}: x_{2}>0\right\} \text {. }
$$

\section{B. Equilibrium Analysis}

From [10], we enumerate the properties of this network. P1: The system (1) has two real equilibria if and only if

$$
E^{2}-4 P r_{1} \geq 0 \Leftrightarrow P \leq \frac{E^{2}}{4 r_{1}} \text {. }
$$

P2: One equilibrium corresponds to a high-voltage/ low-current characteristic, which is stable only if

$$
P \leq \frac{E^{2} C_{1} L_{1} r_{1}}{\left(L_{1}+C_{1} r_{1}^{2}\right)^{2}}
$$

whenever $C_{1}<\left(L_{1} / r_{1}^{2}\right)$.

P3: If $C_{1}>\left(4 L_{1} / r_{1}^{2}\right)$, the strict satisfaction of (2) is sufficient for asymptotic stability of the equilibrium in $\mathrm{P} 2$.

Note that if $P$ is negative, i.e., if the load behaves as a constant power source, then the expressions (2) and (3) are simultaneously satisfied; consequently, this scenario poses no threat regarding voltage collapse nor network's instability, and hence, we focus on the case $P>0 .{ }^{1}$

\section{Objectives and Methodology}

To introduce and support our methodology, we enlist the following remarks [10].

$R$ 1: Observe from $\mathrm{P} 2$ that if the capacitance $C_{1}$ is not big enough, then, in order to maintain the system's stability, the power extraction from the CPL must be strictly smaller than the upper bound for existence of equilibria given in (2).

R2: P3 suggests a passive method to enlarge the domain of values of $P$ for which stability is ensured. It consists in increasing the effective capacitance $C_{1}$, which can be achieved with the open-loop parallel interconnection of a suitable capacitor and the CPL. Some disadvantages of this approach are reviewed in [3, Sec. III.A].

In view of these remarks, we specify our control objectives as follows.

O1: Regulate the voltage $x_{2}$ around a constant value.

O2: Relax the upper bound for $P$ established in (3).

O3: Achieve these objectives without the knowledge of $P$.

Following the work [3] and [16], to achieve these objectives, we add a power converter in parallel with the CPL and design a control strategy that stabilizes the overall network to the desired equilibrium point. The detailed description of the augmented circuit is carried out in Section III and the presentation of the control law-which is the main contribution of this article-is done in Section IV.

\footnotetext{
${ }^{1}$ In the cases in which $P \leq 0$ stabilization may still be desired to improve system damping or to avoid tripping the system due to undervoltage or overvoltage caused by, e.g., disturbances in the source voltage, we refer the reader to [18], where an equilibria analysis is conducted for resistive dc networks containing both constant power sources and loads.
} 


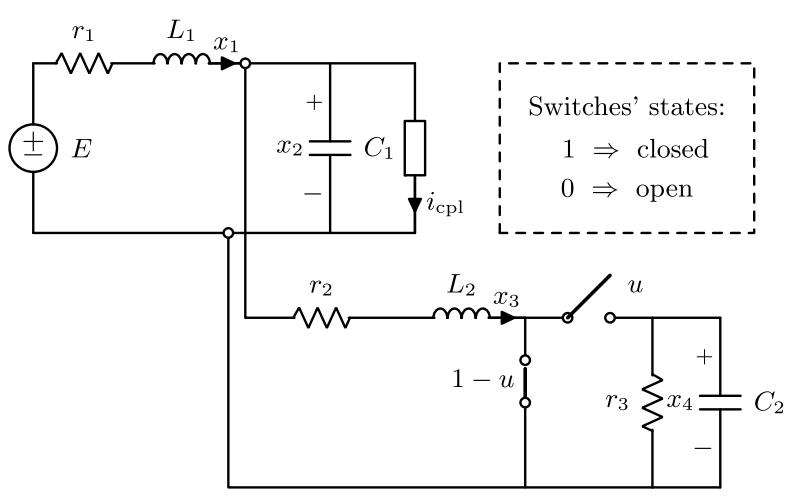

Fig. 2. Network of Fig. 1 is augmented by adding a power converter in parallel with the load.

\section{Augmented Circuit Model}

As proposed in [3] and [16], we augment the network of Fig. 1 by adding a controlled dc-dc power converter in parallel with the load, which results in the circuit shown in Fig. 2. The converter, which in the sequel is referred to as shunt damper, is composed of two complementary switches $u$ and $(1-u)$, a lossy inductor $L_{2}>0$, a capacitor $C_{2}>0$, and a resistor $r_{3}>0$; the latter models the losses associated with the switching devices.

The averaged dynamic model of the augmented system is given by

$$
\begin{aligned}
& L_{1} \dot{x}_{1}=-r_{1} x_{1}-x_{2}+E \\
& C_{1} \dot{x}_{2}=x_{1}-\frac{P}{x_{2}}-x_{3} \\
& L_{2} \dot{x}_{3}=-r_{2} x_{3}+x_{2}-x_{4} u \\
& C_{2} \dot{x}_{4}=-\frac{1}{r_{3}} x_{4}+x_{3} u
\end{aligned}
$$

where $x_{3}$ is the current through $L_{2}, x_{4}$ is the voltage across $C_{2}$, and $u \in(0,1)$ - which is the system's control variablerepresents a duty cycle. The state space of the system is given as

$$
\mathcal{X}:=\left\{x \in \mathbb{R}^{4}: x_{2}>0, x_{4}>0\right\} .
$$

We make the important observations that $x_{1}$-being the current of a reduced model of the network - is not measurable, and the power $P$ is unknown.

For future reference, we write the system (4) in the standard input-affine form, $\dot{x}=f(x)+g(x) u$, by defining

$$
f(x):=\left[\begin{array}{c}
-\frac{r_{1}}{L_{1}} x_{1}-\frac{x_{2}}{L_{1}}+\frac{E}{L_{1}} \\
\frac{x_{1}}{C_{1}}-\frac{P}{C_{1} x_{2}}-\frac{x_{3}}{C_{1}} \\
-\frac{r_{2}}{L_{2}} x_{3}+\frac{x_{2}}{L_{2}} \\
-\frac{1}{r_{3}} \frac{x_{4}}{C_{2}}
\end{array}\right], \quad g(x):=\left[\begin{array}{c}
0 \\
0 \\
-\frac{x_{4}}{L_{2}} \\
\frac{x_{3}}{C_{2}}
\end{array}\right]
$$

the $i$ th entries of which are denoted as $f_{i}$ or $g_{i}$.

\section{Main Results}

In this section, we propose a nonlinear, adaptive, statefeedback controller that is such that the augmented network of Fig. 2 complies with the control objectives described in Section II-C. Toward this end, first, we analyze the equilibria of (4) and establish constraints, on the system's parameters, for their physical feasibility. Second, under the assumption that $x_{1}$ can be measured and the constant parameter $P$ is known, we present a full-information input-output linearization controller [7] that asymptotically stabilizes a specified equilibrium state. Finally, this controller is complemented with an observer for $x_{1}$ and an online estimator for $P$-yielding an adaptive, output-feedback controller design.

\section{A. Existence of Equilibria}

A pair $(\bar{x}, \bar{u}) \in \mathcal{X} \times(0,1)$ is an equilibrium of (4) if and only if it belongs to the set

$$
\mathcal{E}:=\{(\bar{x}, \bar{u}) \in \mathcal{X} \times \mathbb{R}: f(\bar{x})+g(\bar{x}) \bar{u}=0\}
$$

where the mappings $f$ and $g$ are given in (5). An important issue in the design is to ensure that the power consumption of the shunt damper is minimal. In order to carry-out this analysis, we find convenient to parameterize $\bar{x}$ in terms of $\bar{u}$ as follows.

Proposition 1: Consider the augmented circuit model (4). Fix $0<\bar{u}<1$ as the desired steady state duty cycle. The following conditions hold true.

$C l:(\bar{x}, \bar{u}) \in \mathcal{E}$ if and only if

$$
\Delta(P):=E^{2} \ell_{2}-4 \operatorname{Pr}_{1} \ell_{1} \geq 0
$$

where

$$
\ell_{1}:=r_{3} \bar{u}^{2}+r_{1}+r_{2}, \quad \ell_{2}:=r_{3} \bar{u}^{2}+r_{2}
$$

and

$$
\begin{aligned}
& \bar{x}_{1}=\frac{1}{2 r_{1} \ell_{1}}\left[E\left(\ell_{2}+2 r_{1}\right)-\sqrt{\ell_{2}} \sqrt{\Delta(P)}\right] \\
& \bar{x}_{2}=\frac{1}{2 \ell_{1}}\left[\sqrt{\ell_{2}} \sqrt{\Delta(P)}+E \ell_{2}\right] \\
& \bar{x}_{3}=\frac{1}{2 \ell_{1}}\left(\frac{\sqrt{\Delta(P)}}{\sqrt{\ell_{2}}}+E\right) \\
& \bar{x}_{4}=\frac{r_{3} \bar{u}}{2 \ell_{1}}\left(\frac{\sqrt{\Delta(P)}}{\sqrt{\ell_{2}}}+E\right) .
\end{aligned}
$$

C2: The power dissipated at the shunt damper in steady state, i.e., the quantity

$$
\begin{aligned}
\mathcal{P}_{\mathrm{L}}(P) & :=r_{2} \bar{x}_{3}^{2}+\frac{1}{r_{3}} \bar{x}_{4}^{2}, \\
& =\frac{1}{4 \ell_{1}^{2}}(\underbrace{r_{2}+r_{3} \bar{u}^{2}}_{\ell_{2}})\left(\frac{\sqrt{\Delta(P)}}{\sqrt{\ell_{2}}}+E\right)^{2}
\end{aligned}
$$

attains a maximum at $P=0$ if and only if $\Delta(P)>0$.

Proof: The proof of the first claim follows from straightforward algebraic calculations. For the second claim, we compute

$$
\frac{d \mathcal{P}_{\mathrm{L}}(P)}{d P}=-\frac{r_{1} \sqrt{\ell_{2}}}{\ell_{1} \sqrt{\Delta(P)}}\left(\frac{\sqrt{\Delta(P)}}{\sqrt{\ell_{2}}}+E\right)
$$


which is strictly negative if and only if $\Delta(P)>0$, implying that $\mathcal{P}_{\mathrm{L}}(P)$ is monotonically decreasing in its domain of out $\left[0,\left(\ell_{2} / \ell_{1}\right)\left(E^{2} / 4 r_{1}\right)\right]$, hence the claim.

Note that condition (6) is equivalent to

$$
P \leq \frac{\ell_{2}}{\ell_{1}} \frac{E^{2}}{4 r_{1}}
$$

which is more restrictive than the necessary and sufficient condition given in (2) for the existence of equilibria of the dc network without the shunt damper (see Fig. 1) since $\left(\ell_{2} / \ell_{1}\right)<1$. In Proposition 5, we will establish that, through the proposed control scheme, the equilibrium $\bar{x}$ can be rendered (locally) asymptotically stable, provided that it exists, i.e., as long as (8) is fulfilled; observe that no further conditions on $P$ are imposed therein. Then, the constraint (8) becomes a necessary and sufficient condition for the existence and asymptotic stability of $\bar{x}$. In Lemma 1 , we show that, under certain conditions on the system parameters, $\bar{u}$ can be chosen such that (8) is less restrictive than the stability constraint (3) for the network without the shunt damper.

Lemma 1: Assume that $C_{1}<\left(L_{1} / r_{1}^{2}\right)$. Then, the constraint (8) is less restrictive than (3) if either one of the following conditions is satisfied:

1) $\left(4 C_{1} L_{1} r_{1}^{3} /\left(L_{1}-C_{1} r_{1}^{2}\right)^{2}\right) \leq r_{2}$ and $0<\bar{u}<1$.

2) $\left(4 C_{1} L_{1} r_{1}^{3} /\left(L_{1}-C_{1} r_{1}^{2}\right)^{2}\right)-r_{3}<r_{2}<\left(4 C_{1} L_{1} r_{1}^{3} /\left(L_{1}-\right.\right.$ $\left.\left.C_{1} r_{1}^{2}\right)^{2}\right)$ and $\left(\left(4 C_{1} L_{1} r_{1}^{3}-\left(L_{1}-C_{1} r_{1}^{2}\right)^{2} r_{2}\right) /\left(L_{1}-\right.\right.$ $\left.\left.C_{1} r_{1}^{2}\right)^{2} r_{3}\right)^{1 / 2}<\bar{u}<1$.

Proof: We consider that (8) is less restrictive than (3) if and only if

$$
\frac{E^{2} C_{1} L_{1} r_{1}}{\left(L_{1}+C_{1} r_{1}^{2}\right)^{2}}<\frac{\ell_{2}}{\ell_{1}} \frac{E^{2}}{4 r_{1}}=\frac{r_{3} \bar{u}^{2}+r_{2}}{r_{3} \bar{u}^{2}+r_{1}+r_{2}} \frac{E^{2}}{4 r_{1}} .
$$

The latter inequality is equivalent to

$$
\left(L_{1}-C_{1} r_{1}^{2}\right)^{2} r_{3} \bar{u}^{2}+r_{2}\left(L_{1}-C_{1} r_{1}^{2}\right)^{2}-4 C_{1} L_{1} r_{1}^{3}>0 .
$$

On the one hand, we observe that if $r_{2}\left(L_{1}-C_{1} r_{1}^{2}\right)^{2}-$ $4 C_{1} L_{1} r_{1}^{3} \geq 0$, or equivalently, $4 C_{1} L_{1} r_{1}^{3} /\left(L_{1}-C_{1} r_{1}^{2}\right)^{2} \leq r_{2}$, then (9) is satisfied if $0<\bar{u}<1$. Hence, the conditions in 1) are sufficient for (9) to hold.

On the other hand, if $r_{2}\left(L_{1}-C_{1} r_{1}^{2}\right)^{2}-4 C_{1} L_{1} r_{1}^{3}<0$, or equivalently, $r_{2}<\left(4 C_{1} L_{1} r_{1}^{3} /\left(L_{1}-C_{1} r_{1}^{2}\right)^{2}\right)$, then (9) would be satisfied if $\left(\left(4 C_{1} L_{1} r_{1}^{3}-\left(L_{1}-C_{1} r_{1}^{2}\right)^{2} r_{2}\right) /\right.$ $\left.\left(L_{1}-C_{1} r_{1}^{2}\right)^{2} r_{3}\right)^{1 / 2}<\bar{u}$. The constraint $\left(4 C_{1} L_{1} r_{1}^{3} /\right.$ $\left.\left(L_{1}-C_{1} r_{1}^{2}\right)^{2}\right)-r_{3}<r_{2}$ guarantees that $\bar{u}$ can be smaller than one. Thus, the conditions in 2) are sufficient for (8) to be less restrictive than $(3)$.

In [10], the equilibria $(\bar{x}, \bar{u}) \in \mathcal{E}$ are parameterized in terms of $\bar{x}_{2}$, not in terms of $\bar{u}$, as follows:

$$
\begin{aligned}
& \bar{x}_{1}=\frac{E-\bar{x}_{2}}{r_{1}} \\
& \bar{x}_{3}=-\frac{P r_{1}-E \bar{x}_{2}+\bar{x}_{2}^{2}}{r_{1} \bar{x}_{2}} \\
& \bar{x}_{4}=\frac{1}{r_{1} \bar{x}_{2}} \sqrt{r_{3} \kappa_{1}\left(\bar{x}_{2}, P\right) \kappa_{2}\left(\bar{x}_{2}, P\right)}
\end{aligned}
$$

where

$$
\begin{aligned}
& \kappa_{1}\left(\bar{x}_{2}, P\right):=-\left[P r_{1}+\bar{x}_{2}\left(-E+\bar{x}_{2}\right)\right] \\
& \kappa_{2}\left(\bar{x}_{2}, P\right):=P r_{1} r_{2}+\bar{x}_{2}\left[-E r_{2}+\left(r_{1}+r_{2}\right) \bar{x}_{2}\right] .
\end{aligned}
$$

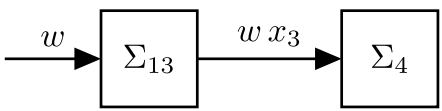

Fig. 3. Block diagram for the cascaded interconnection between the subsystems (11) and (12).

Then, the equilibrium $\bar{x}$, associated with $\bar{x}_{2}=(E / 2)$, is singled out for stabilization. This choice allows the stable operation of the network in a wide range of values of $P$. Unfortunately, the steady-state shunt-damper's power dissipation is given in this case by $E^{2} / 4 r_{1}-P$, which implies a very low energetic efficiency when $P$ is small.

The parameterization that we propose in (7) implies a more involved algebraic expression for the damper's power losses; nonetheless, with an appropriate selection of $\bar{u}$, these losses can be made considerable inferior with respect to the approach adopted in [10]. The effectiveness of this choice is illustrated in the experimental results that are reported in Section VI.

\section{B. Design of a Full-Information Stabilizing Control Law}

In this section, we present a static, state-feedback, control law that renders asymptotically stable the equilibrium point (7). For its design, we assume that $P \geq 0$ is known and fix $\bar{u} \in(0,1)$ such that (6) holds.

Following the ideas presented in [4, Section IV], we introduce the new input:

$$
w=x_{4} u
$$

which allows rewriting the system (4) in the cascade form shown in Fig 3, where

$$
\begin{aligned}
L_{1} \dot{x}_{1} & =-r_{1} x_{1}-x_{2}+E \\
\Sigma_{13}: & C_{1} \dot{x}_{2}=x_{1}-\frac{P}{x_{2}}-x_{3} \\
L_{2} \dot{x}_{3} & =-r_{2} x_{3}+x_{2}-w
\end{aligned}
$$

and

$$
\Sigma_{4}: C_{2} \dot{x}_{4}=-\left(\frac{1}{r_{3}}\right) x_{4}+\frac{1}{x_{4}} x_{3} w .
$$

Proposition 2 presents a control law that ensures voltage regulation and exponential stability of an equilibrium point of $\Sigma_{13}$.

Proposition 2: Fix the constant desired voltage $\bar{x}_{2}>0$. Consider the system (11) in closed loop with the static state feedback

$$
\begin{aligned}
& w\left(x_{1}, x_{2}, x_{3}, P\right) \\
& =-L_{2} C_{1}\left[\beta\left(x_{2}-\bar{x}_{2}\right)+\alpha f_{2}\left(x_{1}, x_{2}, x_{3}\right)\right] \\
& \quad+x_{2}-r_{2} x_{3}-L_{2}\left[f_{1}\left(x_{1}, x_{2}\right)+\frac{P}{x_{2}^{2}} f_{2}\left(x_{1}, x_{2}, x_{3}\right)\right]
\end{aligned}
$$

where $\alpha>0$ and $\beta>0$ are design parameters, and the mappings $f_{i}$ are defined in (5).

Then, the output voltage error

$$
y:=x_{2}-\bar{x}_{2} .
$$


verifies

$$
\ddot{y}+\alpha \dot{y}+\beta y=0
$$

ensuring $\lim _{t \rightarrow \infty} y(t)=0$ (exp.) Moreover, the equilibrium point $\left(\bar{x}_{1}, \bar{x}_{2}, \bar{x}_{3}\right)$ defined in (10) is exponentially stable.

Proof: See Appendix A.

Proposition 3 establishes the stability of the overall system.

Proposition 3: Fix the constant desired voltage $\bar{x}_{2}>0$. Consider the system (11) and (12) in closed loop with the static state feedback (13). Assume that the system's initial condition is sufficiently close to the equilibrium $\bar{x}$ defined in (10). Then, $x_{4}(t)>0$, for all $t \geq 0$ and $\lim _{t \rightarrow \infty} x(t)=\bar{x}$ (exp.).

Proof: See Appendix B.

As a direct application of Propositions 2 and 3, the full-information input-output linearizing controller of the overall dynamics (4) is presented next.

Corollary 1: Consider the system (4) in closed loop with the static state-feedback control law

$$
u=\frac{1}{x_{4}} w\left(x_{1}, x_{2}, x_{3}, P\right)
$$

where $w$ is given in (13). Then, $\bar{x} \in \mathcal{E}$ is a locally, exponentially stable equilibrium point of the closed-loop system.

\section{Output-Feedback Stabilization With Unknown CPL Power}

An adaptive output-feedback version of the controller (16) is proposed now by adding an observer for $x_{1}$ and an online estimator for $P$, which are now assumed to be unmeasured and unknown, respectively.

Proposition 4: Consider the system (1) and assume that $x_{2}$ is positive and belongs to the interval $\left[x_{2}^{\min }, x_{2}^{\max }\right]$. Define the adaptive observer

$$
\begin{aligned}
& \dot{q}_{1}=\frac{E}{L_{1}}-\frac{x_{2}}{L_{1}}-\frac{r_{1}}{L_{1}} \hat{x}_{1}+k_{1} \hat{P}-k_{1} x_{2} \hat{x}_{1}+k_{1} x_{2} x_{3} \\
& \dot{q}_{2}=-k_{2} \hat{P}+k_{2} x_{2} \hat{x}_{1}-k_{2} x_{2} x_{3} \\
& \hat{x}_{1}=q_{1}+\frac{1}{2} k_{1} C_{1} x_{2}^{2} \\
& \hat{P}=q_{2}-\frac{1}{2} k_{2} C_{1} x_{2}^{2}
\end{aligned}
$$

where $k_{1}$ and $k_{2}$ are such that

$$
\begin{aligned}
& 0<k_{1}<8 \frac{k_{2}\left(x_{2}^{\min }+x_{2}^{\max }\right)}{\left(x_{2}^{\max }-x_{2}^{\min }\right)^{2}} \\
& 0<k_{2} .
\end{aligned}
$$

Then

$$
\begin{array}{r}
\lim _{t \rightarrow \infty}\left|\hat{x}_{1}(t)-x_{1}(t)\right|=0 \\
\lim _{t \rightarrow \infty} \hat{P}(t)=P
\end{array}
$$

exponentially.

Proof: See Appendix C.

Remark 1: The design of the adaptive observer in (17) follows the immersion and invariance (I\&I) methodology. The rationale behind this technique, the core mathematical results that define it, as well as its practical application on the adaptive control of electrical systems-including the dc-dc Ćuk and Boost converters - is reported in [17, Ch. 1.3.5, 5, and 8].
TABLE I

PARAMETERS FOR THE CiRCUIT IN Fig. 2

\begin{tabular}{c|c|c|c}
\hline$r_{1}=0.3 \Omega$ & $L_{1}=85.0 \mu \mathrm{H}$ & $C_{1}=200 \mu \mathrm{F}$ & $E=24.0 \mathrm{~V}$ \\
\hline$r_{2}=5 \mathrm{~m} \Omega$ & $L_{2}=100 \mu \mathrm{H}$ & $C_{2}=1.0 \mathrm{mF}$ & $r_{3}=1 \mathrm{k} \Omega$ \\
\hline
\end{tabular}

The stability of the system (4) with an adaptive version of the control law (16) is established next.

Proposition 5: Let $k_{1}$ and $k_{2}$ be such that (18) hold. Fix $\bar{u}$ and compute $\bar{x}$ from (7). Define the output-feedback adaptive control law

$$
u=\frac{1}{x_{4}} w\left(\hat{x}_{1}, x_{2}, x_{3}, \hat{P}\right)
$$

where $w$ is given in (13), and $\hat{x}_{1}$ and $\hat{P}$ are generated by the adaptive observer (17). Then, $\left(x, \hat{x}_{1}, \hat{P}\right)=\left(\bar{x}, \bar{x}_{1}, P\right)$ is an asymptotically stable equilibrium point of the overall system.

Proof: See Appendix D.

Remark 2: A key extension with respect to the research in [10] is the use of an adaptive observer-based feedback law, the implementation of which requires only the measurement of $x_{2}$ and $x_{3}$, which is easy to obtain in a physical setup.

Remark 3: The computation of the control law (13) and (19) requires the knowledge of $\bar{x}_{2}$, which is dependent on the unknown $P$ [see (7)]. In the experiments reported in Section VI, the value of $\bar{x}_{2}$ is computed from the estimate of $P$, i.e. $\hat{P}$, discretely, not continuously in time. This approach is common in hierarchical and supervisory control of ac and dc microgrids [6] and prevents introducing $\bar{x}_{2}$ to the controller if an overshoot of the time-varying signal $\hat{P}$ occurs, and it also simplifies the controller design.

\section{Numerical Simulations}

In this section, we present two simulations to illustrate the performance of the proposed controller. The physical parameters of the system (4) are given in Table I, and the parameters for the adaptive output-feedback controller of (13), (17), and (19) are selected as

$$
\alpha=3 \times 10^{4}, \quad \beta=\frac{\alpha^{2}}{4}, k_{1}=10, k_{2}=10^{4} .
$$

In all simulations, a saturation function has been used to keep $u \in(0,1)$.

\section{A. Simulation 1}

We fix $\bar{u}=0.5$ and compute the equilibrium point $\bar{x}$ to be stabilized via (7) for $P=0 .^{2}$ The initial conditions have been fixed at that equilibrium point. Then, at $t=3 \mathrm{~s}$, a step change in the CPL power, from $P=0 \mathrm{~W}$ to $P=479 \mathrm{~W}$, is introduced, and this yields a new equilibrium point to be stabilized: $\left.\bar{x}\right|_{P=479}$. In addition, at $t=6 \mathrm{~s}$, the CPL is stepped down to $0 \mathrm{~W}$ once again. We note that $P=479 \mathrm{~W}$ verifies

\footnotetext{
${ }^{2}$ By fixing $\bar{u}=0.5$, from Proposition 1 , bullet $\mathrm{C} 2$, we get that $\max \left\{\mathcal{P}_{\mathrm{L}}\right\}=$ $2.29 \mathrm{~W}$, which is attained when $P=0$. Moreover, the maximum value for the CPL's power for which the controlled network admits an equilibrium, obtained from inequality (6), is approximately $479.82 \mathrm{~W}$. These values represent, respectively, $0.47 \%$ and $99.96 \%$ of the maximum power for which the network, without the shunt damper, admits an equilibrium [see inequality (2)].
} 

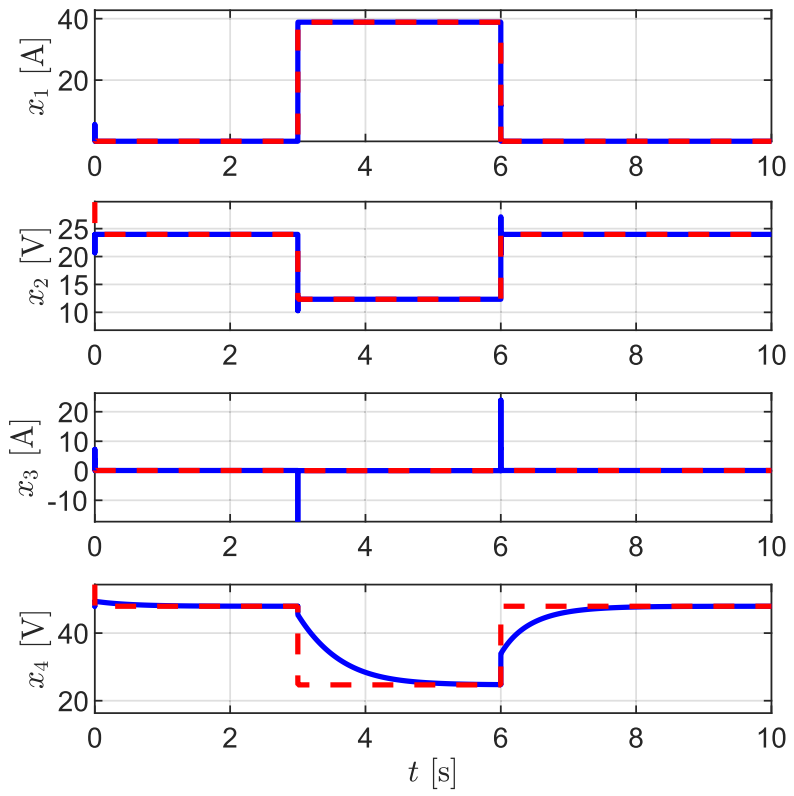

Fig. 4. Time history of the components of $x$ (blue curves) against their reference values (in red dashed line) for Simulation 1. Convergence is achieved despite the step change in the CPL's power.

the new necessary and sufficient condition for the existence and asymptotic stability of an equilibrium (see Proposition 1 and the discussion thereafter). Moreover, it is well above the upper bound for stability given in (3), which reads as follows:

$$
\frac{E^{2} C_{1} L_{1} r_{1}}{\left(L_{1}+C_{1} r_{1}^{2}\right)^{2}}=276.9 \mathrm{~W} \text {. }
$$

Hence, without the shunt damper, the equilibria are unstable.

In Fig. 4, we show the time history of each component of the state $x$ against their equilibrium values; a zoom of these plots around the second transient is shown in Fig. 5. It is clear from these figures that fast convergence is achieved. In Figs. 6 and 7, we plot the control variable $u$. From these figures, we see that, except for some peaks at the instants of power change where the saturation enters into play, the control signal remains all the time within the admissible bounds. In Fig. 6, we also plot the time histories of the estimates $\hat{x}_{1}$ and $\hat{P}$ against their respective reference values, where a fast convergence is evident. Moreover, the plots of $u, \tilde{P}, \tilde{x}_{1}$, and the shunt damper's - very low-power consumption $\mathcal{P}_{\mathrm{L}}$, zoomed around the second transient, are shown in Fig. 7.

\section{B. Simulation 2}

This simulation shows in detail the performance of the adaptive observer. The simulation starts from the initial condition $x(0)=\left.\bar{x}\right|_{P=10}$, and then, at $t=3 \mathrm{~s}$, a large step change in the CPL power, from $P=10 \mathrm{~W}$ to $P=300 \mathrm{~W}$, is introduced. In Figs. 8 and 9, we show the excellent performance of the state observer and the slightly slower tracking of the power estimator.

\section{EXPERIMENTAL VALIDATION}

In order to investigate the practical feasibility of the proposed control scheme, an experimental setup has been
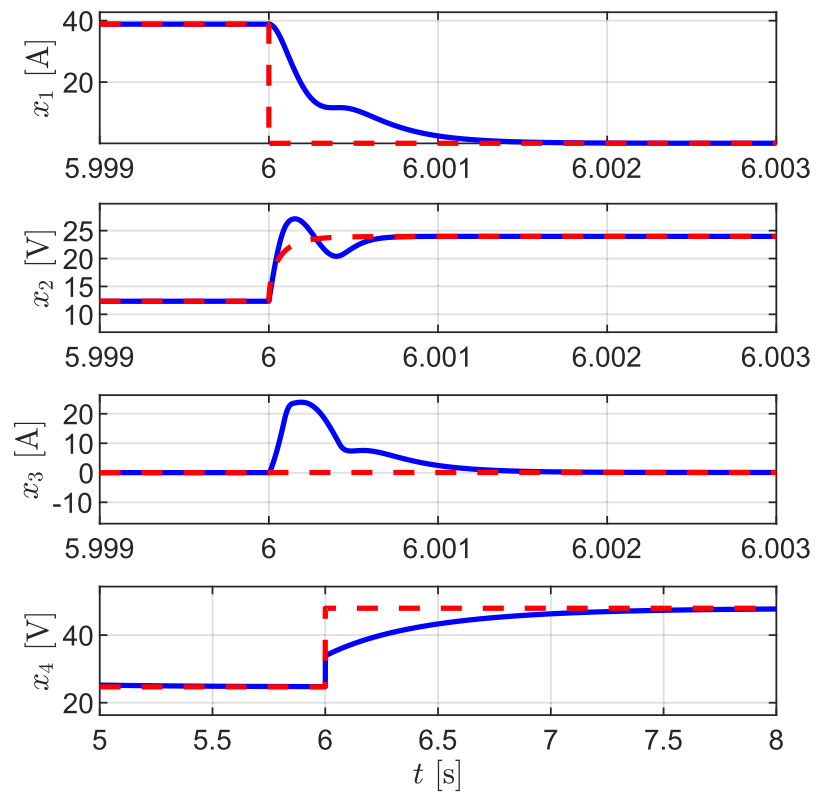

Fig. 5. Zoom around the second transient of the plots in Fig. 4.
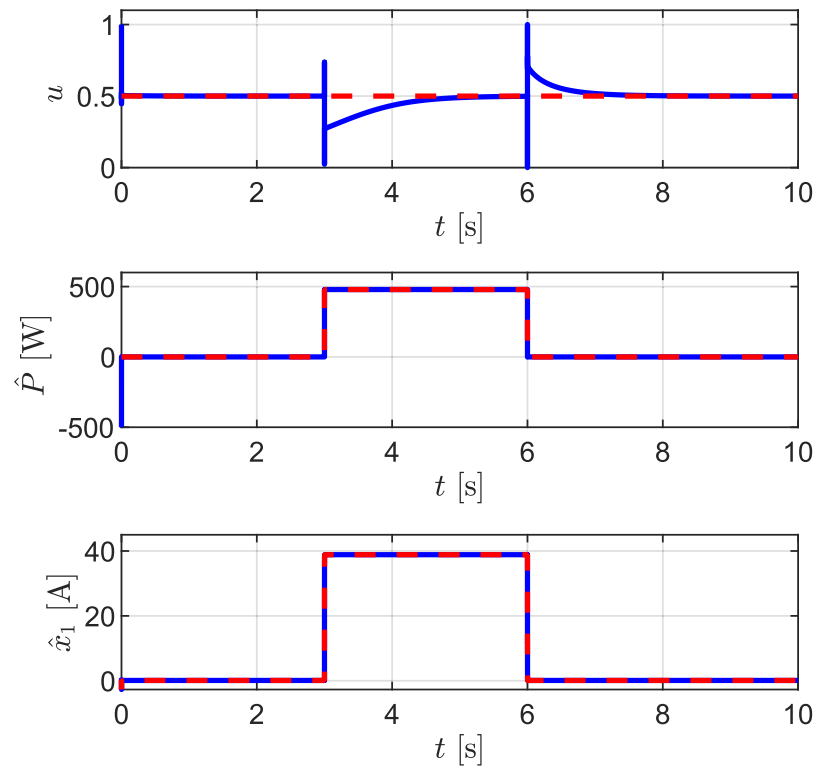

Fig. 6. Time history of $u$, the power estimation $\hat{P}$, and the current observer $\hat{x}_{1}$ (blue line) and their respective reference values (red dashed line) for Simulation 1 .

designed and built according to the electrical scheme shown in Fig. 2. The test bench is composed of a dc voltage source Delta Elektronika SM-52-AR-60, the passive components of the $R L C$ circuit $\left(r_{1}, L_{1}\right.$, and $\left.C_{1}\right)$, a dc boost converter, and a custom-designed and built CPL. The CPL has a maximum allowed current $I_{\max }=40 \mathrm{~A}$ and a maximum power in steady state of $P_{\max }=900 \mathrm{~W}$. The physical setup of the experiments is shown in Fig. 10.

The measured parameters of the system are the same as in Table I, except for $r_{1}$ that in the physical setup is $r_{1}=0.314 \Omega$. The gains of the adaptive output-feedback controller are the same as in Section V. 

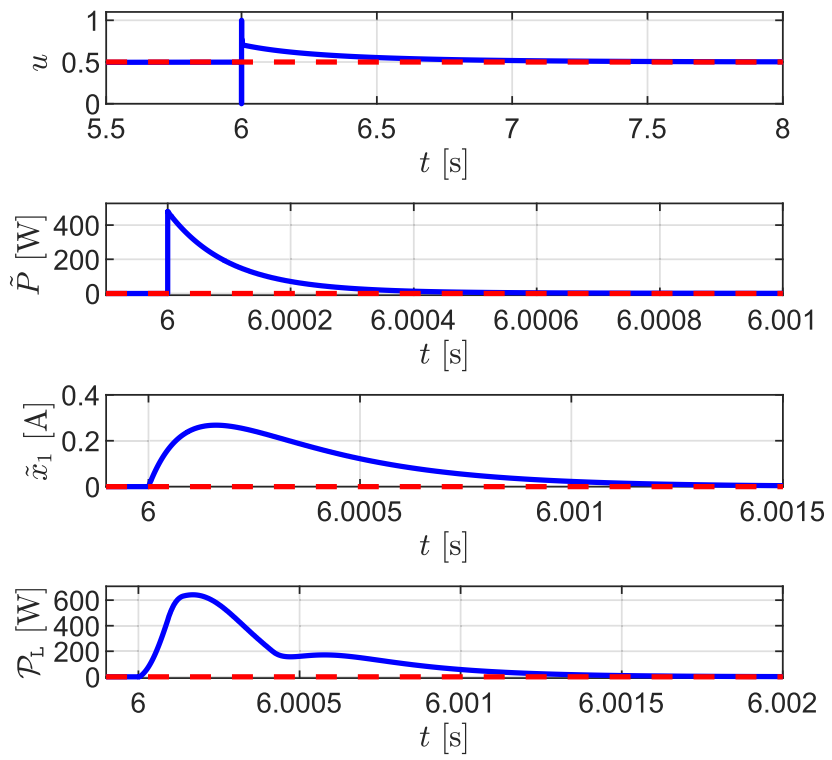

Fig. 7. Zoom around the second transient of the plots in Fig. 6.
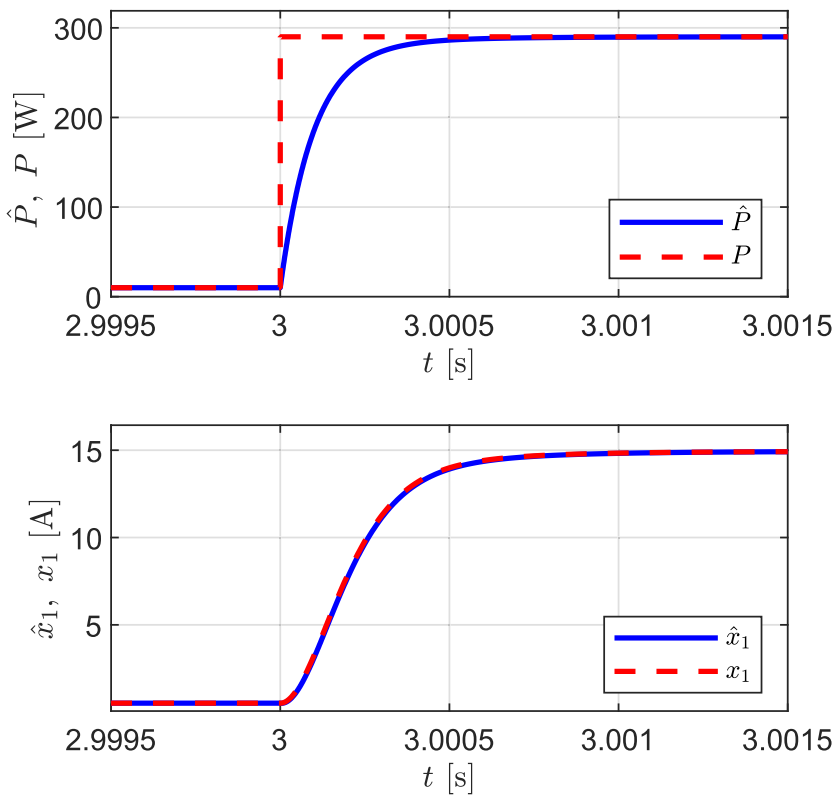

Fig. 8. Time history of $\hat{x}_{1}$ and $\hat{P}$ (blue line) against their respective reference values, $x_{1}$ and $P$ (red dashed line), in Simulation 2. Fast convergence is achieved after the step change in the CPL's power.

It is underscored that the CPL has been designed to approximate an ideal algebraic behavior, i.e., with a much wider bandwidth than the expected dynamics of the whole system. In particular, in a LTspice simulation with an ideal dc voltage source, the CPL settling time for a power step change is $t_{s}=7 \mu \mathrm{s}$. In an experimental test, with the CPL directly connected to the dc voltage source, the time response to a power step change is underdamped, with an approximate settling time of $t_{s}=75 \mu \mathrm{s}$. This change in the load dynamics stems from the nonideal behavior of the dc voltage source, particularly due to its controlled output impedance.
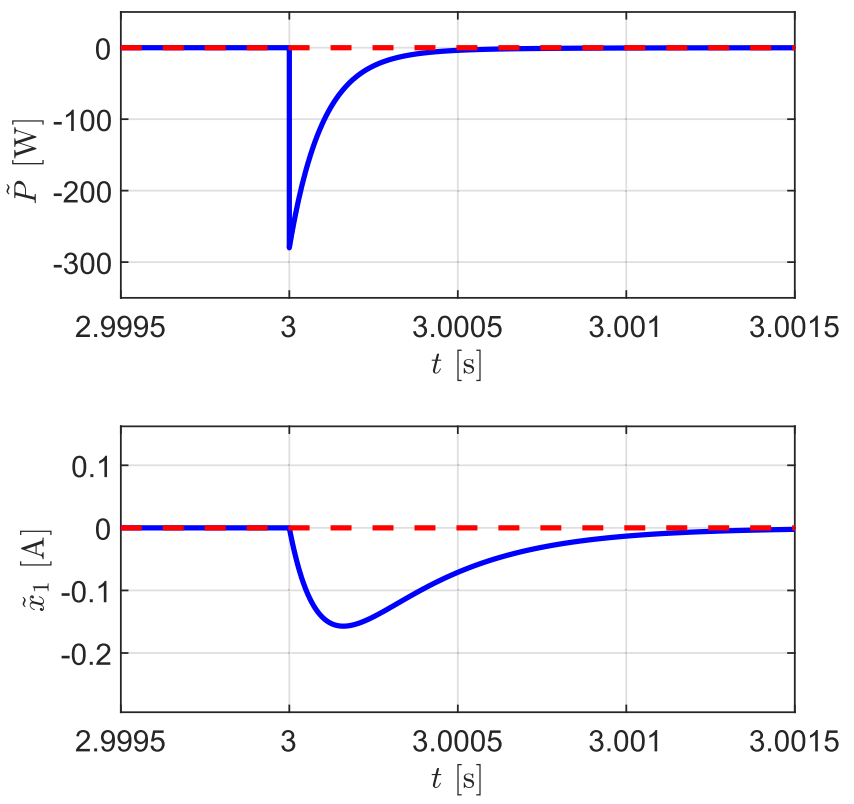

Fig. 9. Zoom around the transient, now of the estimation errors, of the plots in Fig. 8.

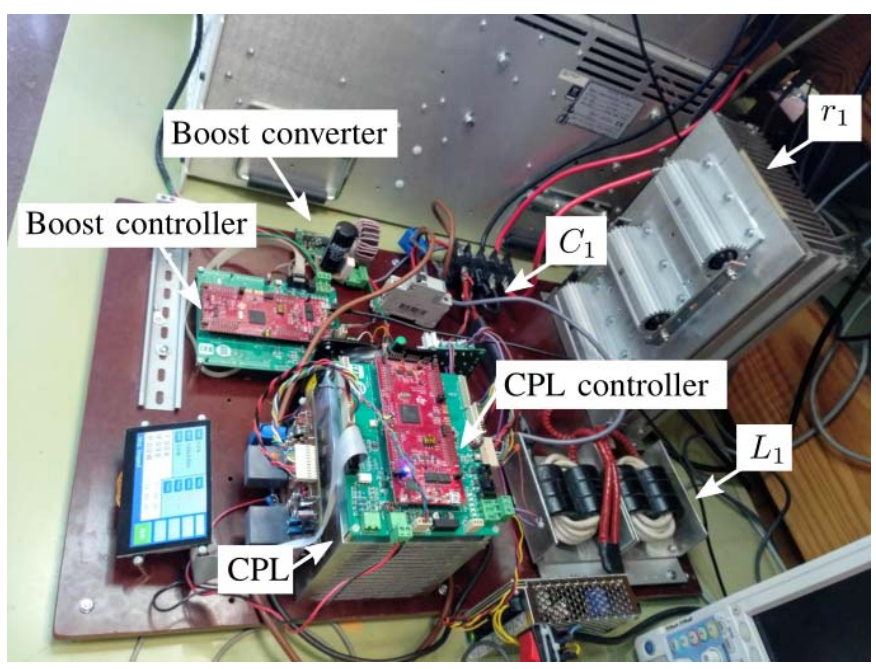

Fig. 10. Experimental test bench. The dc voltage source is not shown here.

The adaptive control law of (19) has been discretized in time using the bilinear transformation (trapezoidal integration) and implemented in a digital signal processor (DSP) TI TMS320F28379D using the automatic code generation tools of Simulink. ${ }^{3}$

\section{A. Experiment 1: System Without the Shunt Damper}

The electrical system without shunt damper is tested first. The objective of this experiment is twofold: 1) to analyze the maximum power that can be demanded by the CPL before the system turns unstable and 2) to illustrate a reduced region of attraction with respect to the system with the controlled shunt damper.

\footnotetext{
${ }^{3}$ The algebraic loops that appear in the equations after the discretization in time have been solved analytically before doing the automatic code generation.
} 


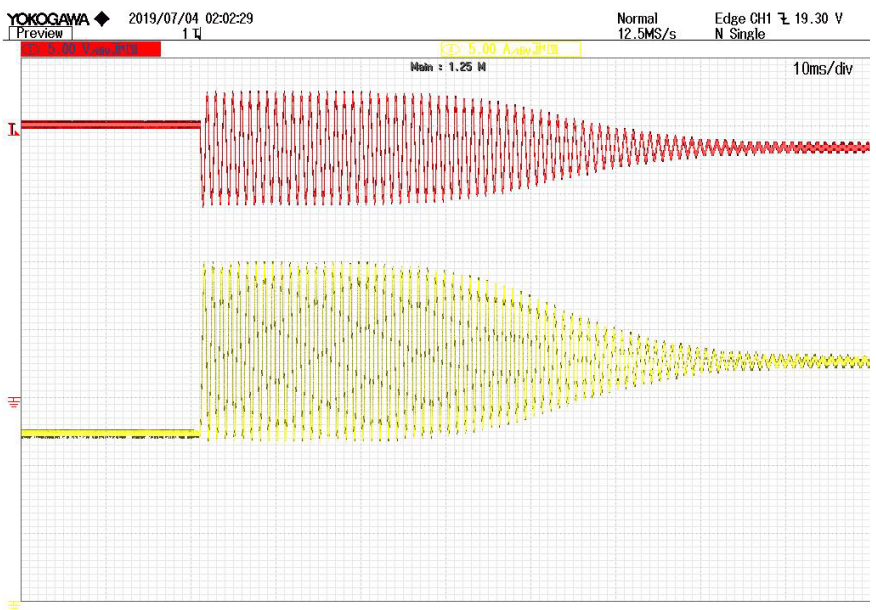

Fig. 11. System response to a step from $P=250 \mathrm{~W}$ to $P=328 \mathrm{~W}$ without the controlled shunt damper. Red curve (top) corresponds to the voltage $x_{2}$ ( $5 \mathrm{~V} / \mathrm{div})$ and yellow curve (bottom) corresponds to the current $x_{1}(5 \mathrm{~A} / \mathrm{div})$. Time scale is $10 \mathrm{~ms} / \mathrm{div}$.

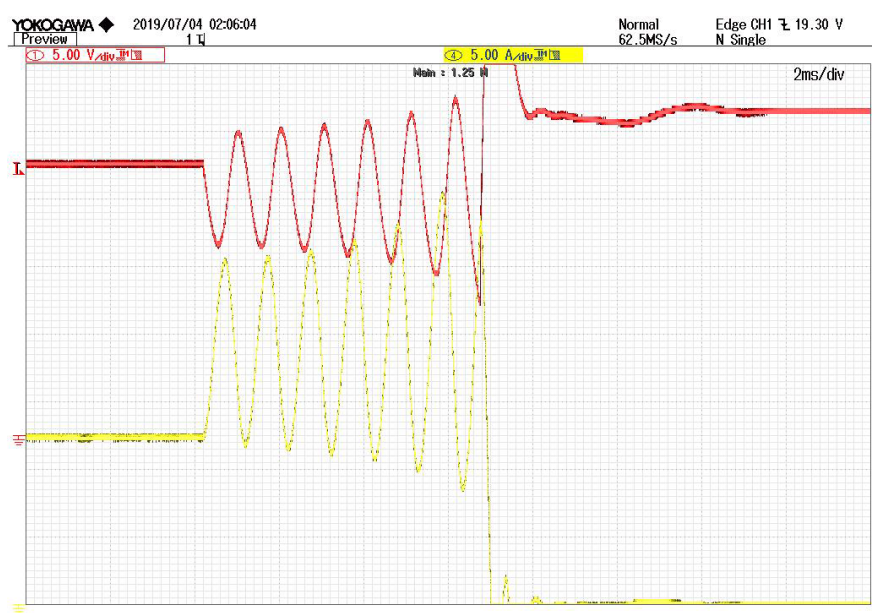

Fig. 12. System response to a step from $P=250 \mathrm{~W}$ to $P=330 \mathrm{~W}$ without the controlled shunt damper. Red curve (top) corresponds to the voltage $x_{2}$ (5 V/div) and yellow curve (bottom) corresponds to the current $x_{1}(5 \mathrm{~A} / \mathrm{div})$. Time scale is $2 \mathrm{~ms} / \mathrm{div}$.

In Fig. 11, we show the response of the system without the shunt damper to a step change of the load's power from $P=250 \mathrm{~W}$ to $P=328 \mathrm{~W}$. The behavior is highly oscillatory but stable, contradicting the predicted necessary bound for stability that is presented in (3), which reads as $P<276.9 \mathrm{~W}$. This discrepancy is explained due to the finite bandwidth of the real CPL.

In Fig. 12, the system response is shown for a step change of the CPL from $P=250 \mathrm{~W}$ to $P=330 \mathrm{~W}$, where an unstable behavior is observed. ${ }^{4}$ It is then concluded that the upper bound for the system's stability is within the interval $[328,330] \mathrm{W}$.

\footnotetext{
${ }^{4}$ In all the unstable experiments, the CPL stops, i.e., abruptly goes to zero, either because the current goes outside of the operating range, which we recall is $[0,40] \mathrm{A}$, or because the voltage at the CPL input terminals falls below the safety limit, which has been situated at a value considerably lower than that corresponding to the maximum power $(\approx 12 \mathrm{~V})$.
}

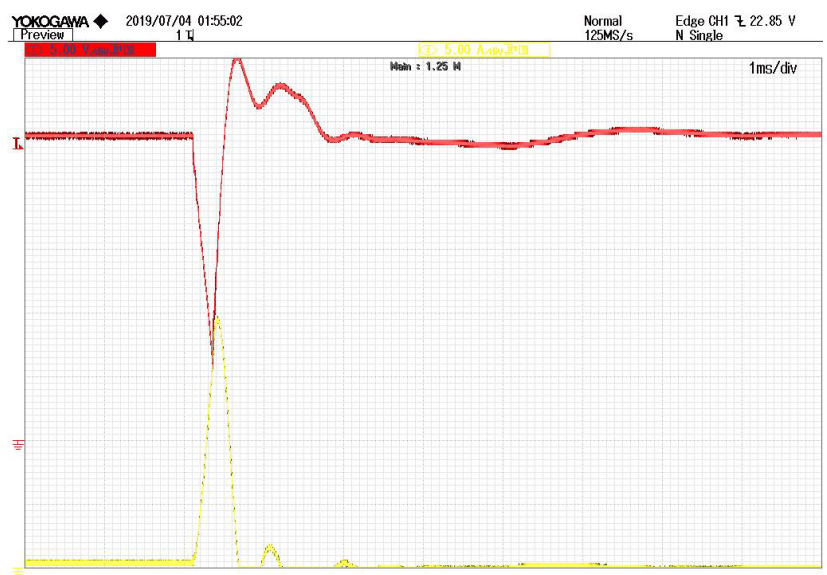

Fig. 13. System response to a step from $P=10 \mathrm{~W}$ to $P=300 \mathrm{~W}$ without the controlled shunt damper. Red curve (top) corresponds to the voltage $x_{2}$ (5 V/div) and yellow curve (bottom) corresponds to the current $x_{1}(5 \mathrm{~A} / \mathrm{div})$. Time scale is $1 \mathrm{~ms} / \mathrm{div}$.

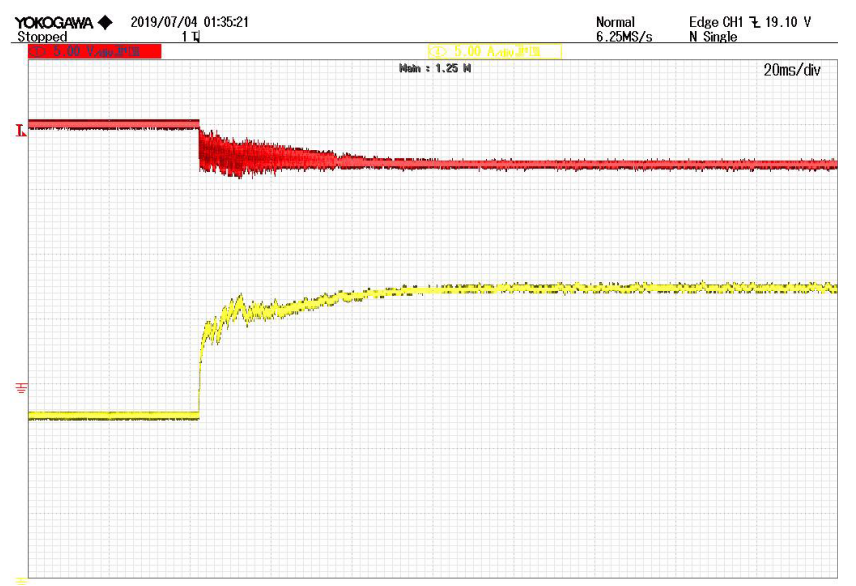

Fig. 14. System response to a step from $P=250 \mathrm{~W}$ to $P=380 \mathrm{~W}$ with the controlled shunt damper. Red curve (top) corresponds to the voltage $x_{2}$ (5 V/div) and yellow curve (bottom) corresponds to the current $x_{1}(5 \mathrm{~A} / \mathrm{div})$. Time scale is $20 \mathrm{~ms} / \mathrm{div}$.

The unstable behavior of the system without the shunt damper to a power step change from $P=10 \mathrm{~W}$ to $P=300 \mathrm{~W}$ can be observed in Fig. 13. Note that even if the load's power is lower than the experimentally determined upper bound for stability, a drastic load change can also destabilize the system. Clearly, in this scenario, the former system's equilibrium lies outside the domain of attraction of the latter one. This behavior is to be contrasted with respect to the operation with the controlled shunt damper discussed next.

\section{B. Experiment 2: System With the Controlled Shunt Damper}

The stable response of the system with the controlled shunt damper in the presence of a step change in the CPL's power, from $P=250 \mathrm{~W}$ to $P=380 \mathrm{~W}$, is shown in Fig. 14. This behavior supports our theoretical and simulation predictions about the system's ability to operate in a stable manner with the proposed control scheme and for values of $P$ beyond the theoretically and experimentally determined upper bounds for 


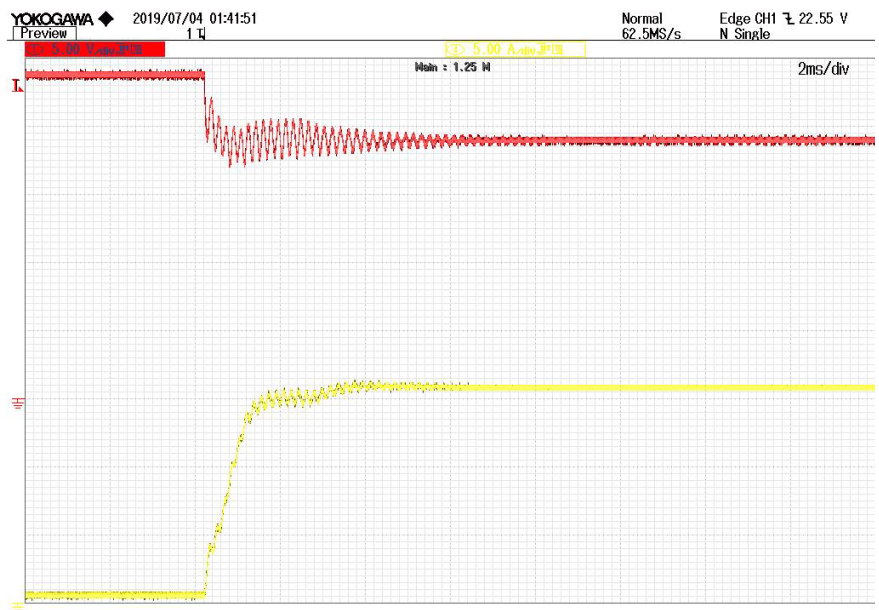

Fig. 15. System response to a step from $P=10 \mathrm{~W}$ to $P=300 \mathrm{~W}$ with the controlled shunt damper. Red curve (top) corresponds to the voltage $x_{2}$ (5 V/div) and yellow curve (bottom) corresponds to the current $x_{1}(5 \mathrm{~A} / \mathrm{div})$. Time scale is $2 \mathrm{~ms} / \mathrm{div}$.

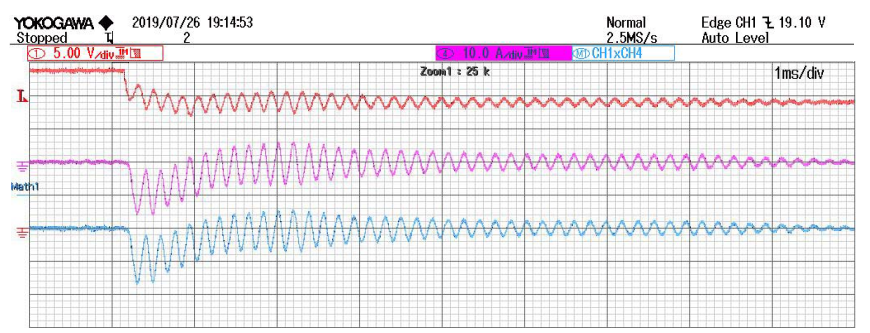

Fig. 16. Measured power consumption of the shunt damper. The system with the controlled shunt damper is subject to a step change in the load's power from $P=10 \mathrm{~W}$ to $P=300 \mathrm{~W}$. Red curve (top) corresponds to the voltage $x_{2}\left(5 \mathrm{~V} /\right.$ div), purple curve (middle) corresponds to the current $x_{3}(10 \mathrm{~A} / \mathrm{div})$, and blue curve (bottom) corresponds to the instantaneous power $(50 \mathrm{~W} / \mathrm{div})$. Time scale is $1 \mathrm{~ms} / \mathrm{div}$.

stability for the network without the shunt damper, which are approximately $276 \mathrm{~W}$ and $330 \mathrm{~W}$, respectively.

The system response to a CPL step change from $P=10 \mathrm{~W}$ to $P=300 \mathrm{~W}$ is shown in Fig. 15, where a stable behavior is observed. By making a comparison with the results reported in Fig. 13, it can be concluded that the domain of attraction of the system with the controlled shunt damper has been successfully increased with respect to its operation without the shunt damper.

To study the energetic efficiency of the proposed active damping scheme, in Fig. 16, we report the measured power consumption of the shunt damper during its operation and in the presence of a step change in the CPL's power from $P=10 \mathrm{~W}$ to $P=300 \mathrm{~W}$. Clearly, a low power consumption is evidenced. Before the change in the CPL's power, when the system is in steady state, we can appreciate that the power losses are nearly zero (approximately $4 \mathrm{~W}$ ), and these losses are due to the passive components of the converter and the mosfets switching losses. During the first $2 \mathrm{~ms}$ of the transient, the average power is negative, that is, the shunt damper injects power into the system to stabilize it. After the transient, the system reaches another stable equilibrium and the average power losses of the damper return to a minimum.
Finally, from successive experiments with different power steps in the CPL, it has been determined that the experimental limit for the stable operation of the system with the controlled shunt damper is in the range $[410,420] \mathrm{W}$. This value represents, approximately, $90 \%$ of the range of power for existence of equilibrium in the system, which from (2) reads as $P<458.6 \mathrm{~W}^{5}$ Clearly, the former value is drastically superior with respect to the operation of the network without the shunt damper.

\section{CONCLUSION AND FutURE WORK}

In this article, we have proposed a nonlinear stabilization method for a dc small-scale power system supplying electric energy to a CPL. By adding a controlled dc-dc power converter in parallel with the load, we have been able to design, using standard input-output linearization, and a suitably tailored adaptive state observer, a nonlinear output-feedback adaptive control law for the stabilization of the overall network. Furthermore, the proposed design permits the stable operation of the network for a wide range of values of the CPL and is able to relax, under certain conditions, some stability bounds that are imposed if the system were to be operated without the shunt damper. Finally, the good performance of the controller has been validated through experiments on a small-scale de network built in our laboratory.

The results of this article can be extended in the following directions.

1) Explicitly compute estimates for the region of attraction of the equilibrium of the closed-loop system.

2) Theoretically evaluate the robustness, against parameter uncertainty, of the proposed adaptive control.

3) Extend our analysis to multiport networks and with a distributed array of CPLs.

\section{APPENDIX A}

PROOF OF PROPOSITION 2

From (11) a and (14), we have

$$
\dot{y}=\dot{x}_{2}=\frac{1}{C_{1}}\left(x_{1}-\frac{P}{x_{2}}-x_{3}\right)=: \mathcal{F}_{2}\left(x_{1}, x_{2}, x_{3}\right)
$$

and

$$
\ddot{y}=\nabla \mathcal{F}_{2}^{\top}\left[\begin{array}{c}
\frac{1}{L_{1}}\left(-r_{1} x_{1}-x_{2}+E\right) \\
\frac{1}{C_{1}}\left(x_{1}-\frac{P}{x_{2}}-x_{3}\right) \\
\frac{1}{L_{2}}\left(-r_{2} x_{3}+x_{2}-w\right)
\end{array}\right] .
$$

If we substitute $w\left(x_{1}, x_{2}, x_{3}\right)$ from (13) into (20), we get (15), which is clearly an asymptotically stable system.

To show that the equilibrium $\left(\bar{x}_{1}, \bar{x}_{2}, \bar{x}_{3}\right)$ is asymptotically stable, we proceed to analyze the zero dynamics of $\Sigma_{13}$ with output $y$, which is the dynamics of $\Sigma_{13}$ restricted to the set

$$
\begin{aligned}
\mathcal{Z} & :=\left\{\left(x_{1}, x_{2}, x_{3}\right): y=0 \wedge \dot{y}=0\right\} \\
& =\left\{\left(x_{1}, x_{2}, x_{3}\right): x_{2}=\bar{x}_{2} \wedge x_{3}=x_{1}-\frac{P}{\bar{x}_{2}}\right\} .
\end{aligned}
$$

\footnotetext{
${ }^{5}$ Without considering the power needed to supply the operating losses of the boost converter.
} 
Restricting the dynamics of $x_{1}$ to $\mathcal{Z}$ yields

$$
\begin{aligned}
L_{1} \dot{x}_{1} & =-r_{1} x_{1}-\bar{x}_{2}+E \\
& =-r_{1}\left(x_{1}-\bar{x}_{1}\right) .
\end{aligned}
$$

Therefore, $x_{1} \rightarrow \bar{x}_{1}$. Now, in $\mathcal{Z}$, we have that $x_{3}=x_{1}-$ $\left(P / \bar{x}_{2}\right)$, and hence, $x_{3} \rightarrow \bar{x}_{1}-\left(P / \bar{x}_{2}\right)=\bar{x}_{3}$.

\section{APPENDIX B}

\section{ProOF OF PROPOSITION 3}

With the change of coordinates $z=(1 / 2) C_{2} x_{4}^{2}$, the system $\Sigma_{4}$ is equivalent to

$$
\dot{z}=-\frac{2}{r_{3} C_{2}} z+w x_{3} .
$$

From Proposition 2, we have that $\left(\bar{x}_{1}, \bar{x}_{2}, \bar{x}_{3}\right)$ is an exponentially stable equilibrium point of $\Sigma_{13}$ in closed loop with (13). Then, the term $w x_{3}$ remains bounded and converges, exponentially, to the value $\bar{u} \bar{x}_{3} \bar{x}_{4}$. The latter limit is computed from the steady-state expression

$$
-r_{2} \bar{x}_{3}-\bar{u} \bar{x}_{4}+\bar{x}_{2}=0 .
$$

It follows trivially from (21) that:

$$
z \rightarrow \frac{r_{3} C_{2}}{2}\left(w x_{3}\right) \rightarrow \frac{r_{3} C_{2}}{2}\left(\bar{u} \bar{x}_{4} \bar{x}_{3}\right)
$$

at an exponential rate. Using the steady-state expression

$$
\bar{u} \bar{x}_{3}-\frac{1}{r_{3}} \bar{x}_{4}=0
$$

we conclude that any solution $x_{4}$ of $\Sigma_{4}$ is positive for all time and converges exponentially to $\bar{x}_{4}>0$ as long as it starts sufficiently close to $\bar{x}_{4}$.

\section{APPENDIX C}

\section{PROOF OF PROPOSITION 4}

The derivatives of the errors $\tilde{x}_{1}:=\hat{x}_{1}-x_{1}$ and $\tilde{P}:=\hat{P}-P$ are given by

$$
\begin{aligned}
\dot{\tilde{x}}_{1} & =-\frac{r_{1}}{L_{1}} \tilde{x}_{1}-k_{1} x_{2} \tilde{x}_{1}+k_{1} \tilde{P} \\
\dot{\tilde{P}} & =-k_{2} \tilde{P}+k_{2} x_{2} \tilde{x}_{1}
\end{aligned}
$$

which can be equivalently written as

$$
\frac{d}{d t}\left[\begin{array}{c}
\tilde{x}_{1} \\
\tilde{P}
\end{array}\right]=-A\left[\begin{array}{c}
\tilde{x}_{1} \\
\tilde{P}
\end{array}\right]
$$

where

$$
A=\left[\begin{array}{cc}
\frac{r_{1}}{L_{1}}+k_{1} x_{2} & -k_{1} \\
-k_{2} x_{2} & k_{2}
\end{array}\right] .
$$

Next, we show that the origin of the nonautonomous system (23) is exponentially stable. Consider the Lyapunov function candidate

$$
V:=\frac{1}{2}\left[\begin{array}{c}
\tilde{x}_{1} \\
\tilde{P}
\end{array}\right]^{\top} S\left[\begin{array}{c}
\tilde{x}_{1} \\
\tilde{P}
\end{array}\right]
$$

where

$$
S:=\left[\begin{array}{cc}
1 & \frac{k_{1}}{k_{2}} \\
\frac{k_{1}}{k_{2}} & s_{2,2}
\end{array}\right]
$$

and $s_{2,2}$ is a positive constant to be determined. Computing the time derivative of $V$ along the trajectories of system (22) yields

$$
\dot{V}=-\left[\begin{array}{c}
\tilde{x}_{1} \\
\tilde{P}
\end{array}\right]^{\top} \mathcal{M}\left[\begin{array}{c}
\tilde{x}_{1} \\
\tilde{P}
\end{array}\right]
$$

where $\mathcal{M}=1 / 2\left(S A+A^{\top} S\right)$, as shown at the top of the next page. Clearly, $V$ is a strict Lyapunov function for (23) if and only if $\exists \bar{s}_{2,2}$ constant such that $\left.\mathcal{M}\right|_{s_{2,2}=\bar{s}_{2,2}}>0$ and $S>0$ or equivalently

$$
\begin{aligned}
\left.\operatorname{det}(\mathcal{M})\right|_{s_{2,2}=\bar{s}_{2,2}} & >0 \quad \forall x_{2} \in\left[x_{2}^{\min }, x_{2}^{\max }\right] \\
\bar{s}_{2,2} & >\frac{k_{1}^{2}}{k_{2}^{2}}
\end{aligned}
$$

hold simultaneously. To provide evidence of the existence of such $\bar{s}_{2,2}$, we note that $\operatorname{det}(\mathcal{M})$ can be written as a quadratic function of $s_{2,2}$ as follows:

$$
\operatorname{det}(\mathcal{M})=a s_{2,2}^{2}+b s_{2,2}+c
$$

where

$$
\begin{aligned}
& a=-\frac{1}{4} k_{2}^{2} x_{2}^{2} \\
& b=\frac{2 k_{2} r_{1}+k_{1} x_{2}\left(r_{1}+k_{1} L_{1} x_{2}\right)}{2 L_{1}} \\
& c=-\frac{k_{1}^{2}\left(4 k_{2} L_{1} r_{1}+\left(r_{1}+k_{1} L_{1} x_{2}\right)^{2}\right)}{4 k_{2}^{2} L_{1}^{2}} .
\end{aligned}
$$

Due to our assumptions

$$
b^{2}-4 a c=\frac{k_{2} r_{1}^{2}\left(k_{2}+k_{1} x_{2}\right)}{L_{1}^{2}}>0
$$

then

$$
\operatorname{det}(\mathcal{M})=0 \Leftrightarrow s_{2,2} \in\left\{\omega_{1}, \omega_{2}\right\}
$$

where

$$
\begin{aligned}
& \omega_{1}=\frac{-b+\sqrt{b^{2}-4 a c}}{2 a} \\
& \omega_{2}=\frac{-b-\sqrt{b^{2}-4 a c}}{2 a}
\end{aligned}
$$

which satisfy $\omega_{1}<\omega_{2}$. For, if we can find $\bar{s}_{2,2}$, constant, such that

$$
\min \left\{\omega_{1}, \frac{k_{1}^{2}}{k_{2}^{2}}\right\}<\bar{s}_{2,2}<\omega_{2} \quad \forall x_{2} \in\left[x_{2}^{\min }, x_{2}^{\max }\right]
$$

then $V$ would be a Lyapunov function of the error dynamics (23). Indeed, note that $k_{1}^{2} / k_{2}^{2}<\bar{s}_{2,2}$ implies that $V$ is positive definite with respect to $\tilde{x}_{1}=\tilde{P}=0$. Moreover, $\omega_{1}<\bar{s}_{2,2}<\omega_{2}, \forall x_{2} \in\left[x_{2}^{\min }, x_{2}^{\max }\right]$, implies that $\operatorname{det}\left(\left.\mathcal{M}\right|_{s_{2,2}=\bar{s}_{2,2}}\right)>0$ for all $x_{2} \in\left[x_{2}^{\min }, x_{2}^{\max }\right]$, making $V$ monotonically decreasing along solutions of the system (23). 


$$
\begin{aligned}
\mathcal{M} & =\frac{1}{2}\left(S A+A^{\top} S\right) \\
& =\left[\begin{array}{cc}
\frac{r_{1}}{L_{1}} & \frac{1}{2}\left(\frac{k_{1}\left(\frac{r_{1}}{L_{1}}+k_{1} x_{2}\right)}{k_{2}}-k_{2} x_{2} s_{2,2}\right) \\
\frac{1}{2}\left(\frac{k_{1}\left(\frac{r_{1}}{L_{1}}+k_{1} x_{2}\right)}{k_{2}}-k_{2} x_{2} s_{2,2}\right) & -\frac{k_{1}^{2}}{k_{2}}+k_{2} s_{2,2}
\end{array}\right]
\end{aligned}
$$

Now, both $\omega_{1}$ and $\omega_{2}$ can be shown to be monotonically decreasing functions of $x_{2}$, and then

$$
\omega_{1} \leq\left.\omega_{1}\right|_{x_{2}=x_{2}^{\min }} \forall x_{2} \in\left[x_{2}^{\min }, x_{2}^{\max }\right]
$$

and

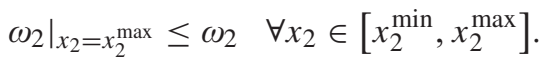

Moreover, the following inequality can be verified through direct algebraic manipulations:

$$
\frac{k_{1}^{2}}{k_{2}^{2}}<\omega_{1} \quad \forall x_{2} \in\left[x_{2}^{\min }, x_{2}^{\max }\right] \text {. }
$$

Under (27)-(29), it is possible to claim that if

$$
\left.\omega_{1}\right|_{x_{2}=x_{2}^{\min }}<\left.\omega_{2}\right|_{x_{2}=x_{2}^{\max }}
$$

then there exists $\bar{s}_{2,2}$ constant such that (26) is satisfied. Through direct computations, it can be verified that a sufficient condition for (30) to hold is given by (18). This concludes the proof. 6

\section{APPENDIX D}

\section{PROOF OF PROPOSITION 5}

Recall from the proof of Proposition 4 that the observer's error dynamics is given by (22). On the other hand, it can be shown through lengthy (but straightforward) computations that (4) in closed loop with (19) has the form

$$
\dot{x}=\left[f(x)+g(x) \frac{w\left(x_{1}, x_{2}, x_{3}, P\right)}{x_{4}}\right]+g(x) \epsilon\left(x, \tilde{x}_{1}, \tilde{P}\right)
$$

where the mapping $\epsilon$ is such that $\epsilon(x, 0,0)=0$ for all $x$.

From Proposition 1, it follows that if $\epsilon \equiv 0$ for all $t$, then $\bar{x}$ is an asymptotically stable equilibrium of (31). Furthermore, Proposition 4 establishes that the origin of the observer's error dynamics is exponentially stable. Consequently, invoking $\left[13\right.$, Proposition 4.1], it is concluded that $\left(x, \tilde{x}_{1}, \tilde{P}\right)=(\bar{x}, 0,0)$ is an asymptotically stable equilibrium point of the overall system.

\footnotetext{
${ }^{6}$ The proofs of inequalities (27)-(29) and the implication $[(18) \Rightarrow(30)]$ have been omitted to enhance readability.
}

\section{REFERENCES}

[1] M. Belkhayat, R. Cooley, and A. Witulski, "Large signal stability criteria for distributed systems with constant power loads," in Proc. Power Electron. Specialist Conf. (PESC), Atlanta, GA, USA, Jun. 1995, pp. $1333-1338$.

[2] M. Belkhayat, R. Cooley, and E. H. Abed, "Stability and dynamics of power systems with regulated converters," in Proc. Int. Symp. Circuits Syst. (ISCAS), Seattle, WA, USA, Apr./May 1995, pp. 143-145.

[3] M. S. Carmeli, D. Forlani, S. Grillo, R. Pinetti, E. Ragaini, and E. Tironi, "A stabilization method for DC networks with constant-power loads," in Proc. IEEE Int. Energy Conf. Exhib. (ENERGYCON), Florence, Italy, Sep. 2012, pp. 617-622.

[4] R. Cisneros, F. Mancilla-David, and R. Ortega, "Passivity-based control of a grid-connected small-scale windmill with limited control authority," IEEE J. Emerg. Sel. Topics Power Electron., vol. 1, no. 4, pp. 247-259, Dec. 2013.

[5] A. Emadi, A. Khaligh, C. H. Rivetta, and G. A. Williamson, "Constant power loads and negative impedance instability in automotive systems: Definition, modeling, stability, and control of power electronic converters and motor drives," IEEE Trans. Veh. Technol., vol. 55, no. 4 pp. 1112-1125, Jul. 2006.

[6] J. M. Guerrero, J. C. Vasquez, J. Matas, L. G. De Vicuna, and M. Castilla, "Hierarchical control of droop-controlled AC and DC microgrids-A general approach toward standardization," IEEE Trans. Ind. Electron., vol. 58, no. 1, pp. 158-172, Jan. 2011.

[7] A. Isidori, Nonlinear Control Systems, 3rd ed. London, U.K.: SpringerVerlag, 1995.

[8] H.-J. Kim, S.-W. Kang, G.-S. Seo, P. Jang, and B.-H. Cho, "Largesignal stability analysis of DC power system with shunt active damper," IEEE Trans. Ind. Electron., vol. 63, no. 10, pp. 6270-6280, Oct. 2016.

[9] J. E. Machado et al., "Active damping of a DC network with a constant power load: An adaptive observer-based design," in Proc. 18th Eur. Control Conf. (ECC), Naples, Italy, Jun. 2019, pp. 411-416.

[10] J. E. Machado, J. Arocas-Pérez, W. He, R. Ortega, and R. Griñó, "Active damping of a dc network with a constant power load: An adaptive passivity-based control approach," in Congreso Nacional de Control Automático (CNCA), San Luis Potosi, Mexico, Oct. 2018, pp. 237-242.

[11] R. D. Middlebrook, "Input filter considerations in design and application of switching regulators," in Proc. IEEE Ind. Appl. Soc. Аnnu. Meeting, Nov. 1976, pp. 366-382.

[12] H. Mosskull, "Optimal DC-link stabilization design," IEEE Trans. Ind. Electron., vol. 62, no. 8, pp. 5031-5044, Aug. 2015.

[13] R. Sepulchre, M. Jankovic, and P. Kokotovic, Constructive Nonlinear Control. London, U.K.: Springer-Verlag, 1997.

[14] S. Singh, A. R. Gautam, and D. Fulwani, "Constant power loads and their effects in DC distributed power systems: A review," Renew. Sustain. Energy Rev., vol. 72, pp. 407-421, May 2017.

[15] M. Wu and D. D.-C. Lu, "A novel stabilization method of LC input filter with constant power loads without load performance compromise in DC microgrids," IEEE Trans. Ind. Electron., vol. 62, no. 7, pp. 4552-4562, Jul. 2015.

[16] X. Zhang, X. Ruan, H. Kim, and C. K. Tse, "Adaptive active capacitor converter for improving stability of cascaded DC power supply system," IEEE Trans. Power Electron., vol. 28, no. 4, pp. 1807-1816, Apr. 2013

[17] A. Astolfi, D. Karagiannis, and R. Ortega, Nonlinear and Adaptive Control With Applications. London, U.K.: Springer-Verlag, 2008.

[18] J. Simpson-Porco, F. Dörfler, and F. Bullo, "On resistive networks of constant-power devices," IEEE Trans. Circuits Syst. II, Exp. Briefs, vol. 62, no. 8, pp. 811-815, Aug. 2015. 


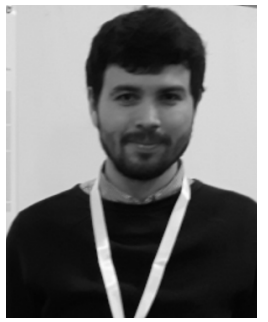

Juan E. Machado received the B.Sc. degree in electromechanical engineering from the Instituto Tecnológico de La Paz, La Paz, Mexico, in 2012, and the M.Sc. degree in applied mathematics from the Centro de Investigación en Matemáticas, Guanajuato, Mexico, in 2015. He successfully defended his $\mathrm{Ph} . \mathrm{D}$. thesis in automatic control from the Universite Paris Sud-CentraleSupélec, Gif-Sur-Yvette, France, in 2019.

He currently holds a post-doctoral position at the Discrete Technology and Production Automation (DTPA) Group, Faculty of Science and Engineering, University of Groningen, Groningen, The Netherlands. His research interest includes modeling and control of electromechanical systems.

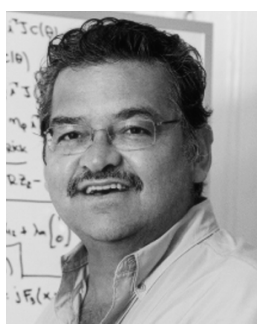

Romeo Ortega was born in Mexico. He received the B.Sc. degree in electrical and mechanical engineering from the National University of Mexico, Mexico City, Mexico, in 1974, the Master of Engineering degree from the Polytechnical Institute of Leningrad, USSR, in 1978, and the Docteur D‘Etat degree from the Polytechnical Institute of Grenoble, Grenoble, France, in 1984.

He then joined the National University of Mexico, where he worked until 1989. He was a Visiting Professor with the University of Illinois at UrbanaChampaign, Champaign, IL, USA, from 1987 to 1988, and McGill University, Montreal, QC, Canada, from 1991 to 1992. He was a member of the French National Research Council (CNRS) from June 1992 to July 2020, where he was a "Directeur de Recherche" at the Laboratoire de Signaux et Systemes (CentraleSupelec), Gif-sur-Yvette, France. He is currently a full-time Professor with the Instituto Tecnológico Autónomo de México (ITAM), Mexico City. He has published five books and more than 350 scientific articles in international journals, with an $\mathrm{H}$-index of 84 . His research interest is in nonlinear and adaptive control, with special emphasis on applications.

Dr. Ortega was a fellow of the Japan Society for Promotion of Science from 1990 to 1991. He has been a fellow of the International Federation of Automatic Control (IFAC) since 2016. He has served as the chairman in several IFAC and IEEE committees and participated in various editorial boards of international journals. He has supervised more than $35 \mathrm{Ph} . \mathrm{D}$. theses. He is also the Editor-in-Chief of International Journal of Adaptive Control and Signal Processing and a Senior Editor of Asian Journal of Control.

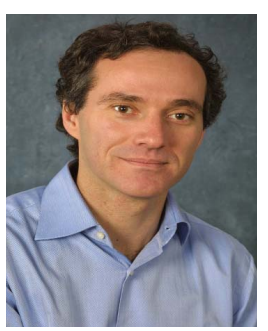

Alessandro Astolfi (Fellow, IEEE) was born in Rome, Italy, in 1967. He graduated in electrical engineering from the University of Rome, Rome, Italy, in 1991. In 1992, he joined ETH-Zurich where he received an M.Sc. degree in information theory in 1995 and the Ph.D. degree with Medal of Honor in 1995 with a thesis on discontinuous stabilization of nonholonomic systems. In 1996, he was awarded a Ph.D. from the University of Rome "La Sapienza" for his work on nonlinear robust control.

In 1992, he joined ETH Zurich. Since 1996, he has been with the Electrical and Electronic Engineering Department, Imperial College London, London, U.K., where he is currently a Professor of nonlinear control theory and the Head of the Control and Power Group. From 1998 to 2003, he was an Associate Professor with the Department of Electronics and Information, Politecnico of Milano, Milan, Italy. Since 2005, he has been a Professor with the Dipartimento di Ingegneria Civile e Ingegneria Informatica, University of Rome "Tor Vergata," Rome. He has been a Visiting Lecturer in nonlinear control in several universities, including ETH Zurich from 1995 to 1996; the Terza University of Rome, Rome, in 1996; Rice University, Houston, TX, USA, in 1999; Kepler University, Linz, Austria, in 2000; Supelec, Paris, France, in 2001, and Northeastern University, Boston, MA, USA, in 2013. He is the author of more than 150 journal articles, 30 book chapters, and over 240 papers in refereed conference proceedings. $\mathrm{He}$ is the author (with D. Karagiannis and R. Ortega) of the monograph Nonlinear and Adaptive Control with Applications (Springer-Verlag). His research interests are focused on mathematical control theory and control applications, with special emphasis on the problems of discontinuous stabilization, robust and adaptive control, observer design, and model reduction.
Dr. Astolfi is a Distinguished Member of the IEEE CSS and a fellow of the International Federation of Automatic Control (IFAC). He was a recipient of the IEEE CSS A. Ruberti Young Researcher Prize in 2007, the IEEE RAS Googol Best New Application Paper Award in 2009, the IEEE CSS George S. Axelby Outstanding Paper Award in 2012, and the Automatica Best Paper Award in 2017. He has served as the Chair for the IEEE CSS Conference Editorial Board from 2010 to 2017 and the IPC of several international conferences. He has been/is a member of the IEEE Fellow Committee in 2016 and from 2019 to 2020. He served as an Associate Editor for Automatica, Systems and Control Letters, the IEEE TRANSACTIONS ON Automatic Control, the International Journal of Control, the European Journal of Control, and the Journal of the Franklin Institute, an Area Editor for the International Journal of Adaptive Control and Signal Processing, a Senior Editor for the IEEE TRAnsactions on Automatic Control, and the Editor-in-Chief for the European Journal of Control. He is also the Editor-in-Chief of the IEEE TRANSACTIONS ON AUtOMATIC CONTROL.

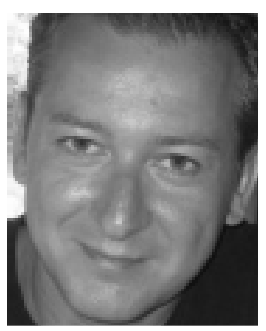

José Arocas-Pérez received the Technical Industrial Engineering degree, the B.Sc. degree in industrial electronics and automation engineering, and the M.Sc. degree in automation and industrial computing, in 2011, 2014, and 2015, respectively, all from the Universitat Politècnica de València (UPV), València, Spain, doing part of his studies at the Instituto Tecnológico de Costa Rica (TEC), Cartago, Costa Rica, with a grant of cooperation. He is currently pursuing the Ph.D. degree with the Advanced Control of Energy Systems Group (ACES), Institute of Industrial and Control Engineering (IOC), Universitat Politècnica de Catalunya (UPC), Barcelona, Spain. The research topic of his doctoral thesis is the development of control techniques for improving stability in power systems with electronic converters working at constant power.

His research activities have focused on automatic control, emulation and simulation of wind power generators, control techniques of power electronic converters, and simulation of biological systems in the field of synthetic biology among others.

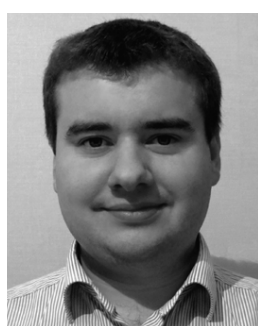

Anton Pyrkin (Member, IEEE) was born in Zaozerniy, USSR, in 1985. He received the B.S., M.S., and Ph.D. degrees and the Doctor of Science (habilitation thesis) degree in system analysis, data processing, and control (in technical systems) from ITMO University, Saint Petersburg, Russia, in 2006, 2008, 2010, and 2015, respectively.

$\mathrm{He}$ is currently a Leading Professor and the Dean of the Faculty of Control Systems and Robotics, ITMO University. He is a coauthor of more than 200 publications in science journals and proceedings of conferences. His research interests include adaptive and robust control, electromechanical systems, robotics, parameter estimation, disturbance cancellation, time-delay systems, nonlinear systems, autopilot, and dynamic position systems for vessels.

Dr. Pyrkin is a member of the International Public Association Academy of Navigation and Motion Control. 


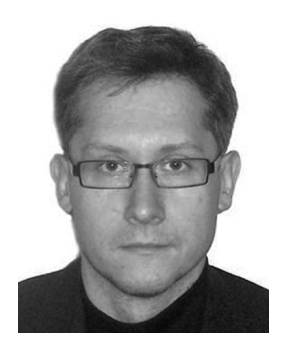

Alexey A. Bobtsov (Senior Member, IEEE) received the M.S. degree in electrical engineering, the Ph.D. and D.Sc. degrees (habilitation thesis) from ITMO University, Saint Petersburg, Russia, in 1996, 1999, and 2007, respectively.

From November 1999 to December 2000, he was an Assistant Lecturer with the Department of Automation and Remote Control. From December 2000 to May 2007, he was an Associate Professor with the Department of Control Systems and Informatics. In May 2007, he was appointed as a Professor at the Department of Control Systems and Informatics. In September 2008, he was elected as the Dean at the Faculty of Computer Technologies and Controlling Systems. He is currently the Dean of the Faculty of Computer Science and Control and the Head of the Department of Control Systems and Informatics, ITMO University. He is the coauthor of more than 200 journals and conference papers, five patents, and 15 books and textbooks. His research interests include nonlinear and adaptive control, control of oscillatory and chaotic systems, and computer-aided control systems design with applications to mechanical and robotic systems.

Dr. Bobtsov is a member of the International Public Association Academy of Navigation and Motion Control.

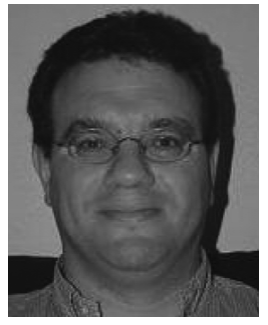

Robert Griñó (Senior Member, IEEE) received the M.Sc. degree in electrical engineering and the Ph.D. degree in automatic control from the Universitat Politecnica de Catalunya (UPC), Barcelona, Spain, in 1989 and 1997, respectively.

From 1990 to 1991, he was a Research Assistant with the Instituto de Cibernética, UPC. From 1992 to 1998, he was an Assistant Professor with the Automatic Control Department, UPC, where he has been an Associate Professor since 1998. He is currently Director of the Institute of Industrial and Control Engineering (IOC), UPC. His research interests include digital control, nonlinear control, and control of power electronic converters.

Dr. Griñó is a member of the Spanish Committee of Automatica (CEA-IFAC) and an affiliate of the International Federation of Automatic Control (IFAC) and a member of its Technical Committee 6.3, "Power and Energy Systems." 